\title{
Performance enhancement of relays used for next generation wireless communication networks
}

\author{
Saraju Prasad Padhy', Madhusmita Panda ${ }^{2}$, Srinivas Sethi ${ }^{3}$, Aruna Tripathy ${ }^{4}$ \\ ${ }^{1}$ Assistant General Manager, Bharat Sanchar Nigam Limited, Gangtok, India \\ ${ }^{2}$ Department of ECE, S 'O' A University, Bhubaneswar, India \\ ${ }^{3}$ Department of CSE \& A, Indira Gandhi Institute of Technology, Sarang, India \\ ${ }^{4}$ Department of Instrumentation and Electronics, College of Engineering and Technology, Bhubaneswar, India
}

\section{Article Info \\ Article history: \\ Received Dec 18, 2019 \\ Revised Apr 29, 2020 \\ Accepted Feb 8, 2021}

\section{Keywords:}

Cooperative relaying

LTE advanced

Optimization

PSO

Relay

\begin{abstract}
Relaying is one of the latest communication technologies developed for wireless networks like WiMAX, LTE Advanced and 5G Ultra Reliable Low Latency Communication (URLLC) networks to provide coverage extension as well as higher bitrates for cell edge users. Thus they are included in the design of next generation wireless communication systems to provide performance improvement in terms of coverage and capacity over their predecessors. Other promising features of this technology include easy to implement and reduction in deployment cost. The objective of this paper is to analyze both cooperative and non-cooperative relaying techniques in the Infinite Block length regime and findout the benefits of Relay implementation. A single Amplify and Forward (A\&F) Relay is used for this purpose. Reduction in power requirement for Simple Relay is shown in comparison to the direct transmission, using experimental analysis with Matlab simulation. SER (Symbol error rate) is calculated at the receiver for no relay, simple Relay and Cooperative Relaying scenario to show the improvements of cooperative Relaying implementation. The performance enhancement of the Relay is then carried out using Particle Swam Optimization (PSO) optimization technique where allocation of power between Base station and Relay Node is effectively distributed for optimum performance.
\end{abstract}

This is an open access article under the CC BY-SA license.

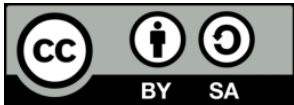

Corresponding Author:

Saraju Prasad Padhy

Assistant General Manager

Bharat Sanchar Nigam Limited

Qr No-5, Type-5, Postal Colony, Bhubaneswar, India

Email: sppadhy71@gmail.com

\section{INTRODUCTION}

As per International Mobile Telecommunication (IMT) standards the $4^{\text {th }}$ generation wireless commu- nication also termed as Long-Term Evolution Advanced (LTE-A) or 4G can support bit rate of 1 Gbps for fixed wireless application or low mobility cases upto $10 \mathrm{kmph}$. It can support bit rate upto 100 Mbps for high mobility environment upto $350 \mathrm{kmph}$. The transmission Bandwidth can go upto to $100 \mathrm{MHz}$ where as spectral efficiency for downlink can be upto $15 \mathrm{~b} / \mathrm{s} / \mathrm{Hz}$ and that of uplink can be upto $6.75 \mathrm{~b} / \mathrm{s} / \mathrm{Hz}$ respectively [1]. Considering average data rate per active subscriber to be $512 \mathrm{kbps}$, number of subscribers per $\mathrm{MHz}$ can be $13(6.75 / 0.5)$ to $30(15 / 0.25)$. To achieve these requirements prescribed by International Telecommunication Union (ITU) LTE-A standardization work has been introduced by the third Generation 
Partnership project (3GPP) which recommended new transmission technologies like Carrier aggregation, coordinated Multipoint transmission (CoMP), Inter-Cell Interference Avoidance (ICIA), Flexible Spectrum Usage (FSU), Higher order Sectorization (HOS), Home e-nodeB (HeNB) implementation \& Relaying [2-4]. During implementation of the network generally it is seen that strength of the signal becomes weak at cell edges, inside buildings or tunnels mainly due to higher frequencies (above $2 \mathrm{GHz}$ ) resulting greater attenuation [5]. So strength of the received signal needs to be increased without increase in the deployment cost.

Relay node (RN) can be implemented as a solution in this regard by placing it in between eNodeB and UE for forwarding information generated by user from UE to eNodeB and vice versa. In order to fulfil better service quality (QoS) and higher data rate, generally density of Base Stations is increased. Although this provides wireless access to far-reached places of the coverage area, network cost increases. Use of RNs enables rapid and cost effective deployment of the network infrastructure [6]. Deployment of RNs not only improves the signal strength for indoor applications but also overcome the coverage limitations at the celledges. Hence Relays are generally placed in coverage holes or cell-edges and can work either as regenerator or amplifier for the signal. Both techniques are quite useful even though they have their advantages and disadvantages. The RNs are connected to the core network via eNodeB using wireless backhaul links as shown in Figure 1.

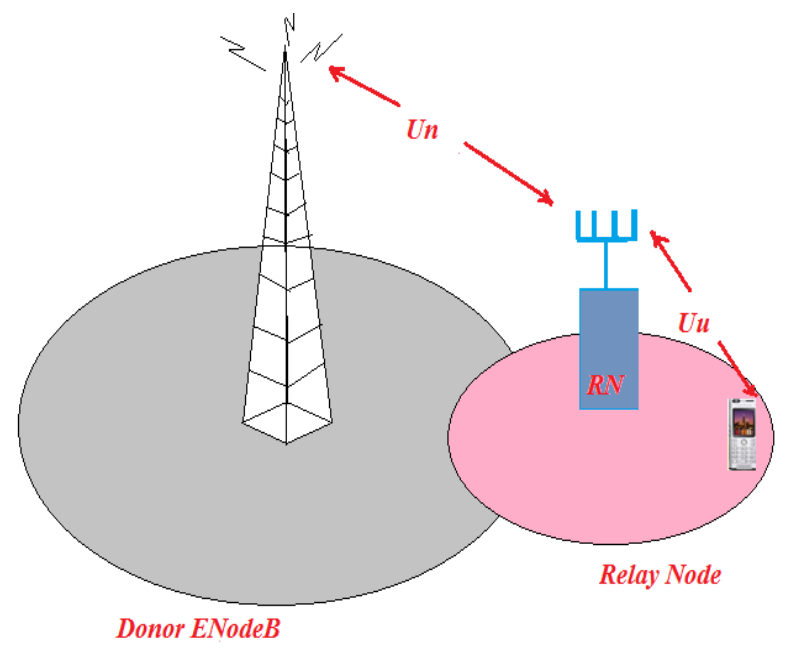

Figure 1. Relay node deployment

\section{OVERVIEW OF RELAY TECHNIQUES}

Generally Relay nodes are classified with reference to their protocol architecture. From that prospective, RNs are classified into three different types: Layer 1 Relay (L1), Layer 2 Relay (L2) and Layer 3 Relay (L3) [3, 7]. Cell coverage can be improved by L1 relay. Delay in this case is the smallest in comparison to L2 and L3 relays. However L1 relays cannot improve the spectral efficiency as physical link amplifies noise. Cell coverage can also be improved by L2 relay. It can also minimise the error on RN-UE and eNodeB-RN links. L3 relays have all the benefits of L2 relay in addition to the fact that it reduces latency.

In 3GPP Release 8, categorization basing upon the protocol architecture has lost its significance for designing RAN (Radio Access Network). Hence conventional L1, L2 and L3 relays were replaced by two different relay types such as Type-I and Type-II which became more relevant [8].While Type-I relay (Nontransparent relay) need to transmit the control information and common reference signal to the eNodeB, Type-II relay (Transparent Relay) doesn't. Further Type-I relay is used for coverage extension where as Type-II relay can be mainly used for enhancement of bitrate as a result of which capacity of system increases. As Type-I Relay transmits both traffic data and control information, more overhead is consumed causing increasesd transmission delay.Type-II relay exchanges control information to and from Base Station directly and only transmits the user data between Base Station \& UE as a result of which performance inefficiency is curbed.

Relays are further classified into THREE different types as per the received signal processing and further transmission [3]. Amply \& Forward (A\&F) Relay forwards the received signal to the destination after 
amplifying it without any further signal processing. Decode and Forward (D\&F) Relay first decodes the received signal from the source node and then encodes the signal before it is being sent to the destination [9]. In the Demodulation and Forward (DM\&F) Relay, received signal is demodulated in the first phase and then a decision is made before modulating and forwarding the newly constructed signal to the destination at the second phase. Among the THREE schemes, A\&F is the one which is simplest and offers low latency. However, as it amplifies noise, the receiving performance degrades. In case of $\mathrm{D} \& \mathrm{~F}$, the error propagation can be avoided even though processing delay is relatively long. However DM\&F offers a trade-off between complexity and performance. The biggest issue with DM\&F Relay is that as the decision is taken at first phase, it becomes difficult to avoid propagation of error.

\section{PERFORMANCE MEASUREMENT OF SIMPLE TWO HOP RELAYING SYSTEM}

Performance of a Simple Relay system is evaluated here using experimental method. The following parameters are considered for the purpose. Values for some of the parameters considered are shown in Table 1. Two different cases are considered for this purpose. In the first instance eNodeB and RN are considered to be fixed nodes and UE is a movable node where as in the second instance eNodeB and UE are fixed nodes where as Relay is the movable node.

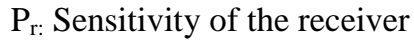

d: Distance of UE from eNodeB

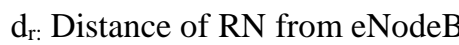

$\mathrm{y}$ : Component for path loss

$\mathrm{P}_{\mathrm{tx}}$ : Transmitted power under no $\mathrm{RN}$

$\mathrm{P}_{\mathrm{tb}}$ : Transmitted power requirement from eNodeB to Relay

$\mathrm{P}_{\mathrm{tr}}$ : Transmitted power requirement from Relay to UE

$\mathrm{P}_{\mathrm{tm}}$ : Total power transmitted required after deployment of Relay Node

Table 1. Parameters and values considered for RN implementation

\begin{tabular}{cc}
\hline Parameter & Value \\
\hline $\mathrm{P}_{\mathrm{r}}$ & $10^{-3}$ \\
$\mathrm{y}$ & 4 \\
$\mathrm{~d}_{\mathrm{r}}$ & 400 \\
\hline
\end{tabular}

\subsection{Calculation of power requirement when eNodeB and RN are fixed nodes and $\mathrm{UE}$ is movable}

The total distance between the eNodeB and UE is divided into two parts. One part is the distance between eNodeB and RN where as the other part is the distance between RN to UE. The sensitivity of RN \& UE are assumed to be the same and fixed. By moving the UE away from eNodeB, we find out the transmitted power requirement between UE \& RN.This is added to the transmitted power requirement between eNodeB and RN to find out the total transmitted power. This value is compared with the transmitted power without relay node considering other parameters to be same.

Algorithm 1: Calculation of optimum power requirement when base station and Relay are fixed nodes and UE is a moving node.

$\mathrm{d}=500: 100: 2000$

for $\mathrm{x}=1$ to 16

$\mathrm{P}_{\mathrm{tx}}(\mathrm{x})=\operatorname{Pr} .[\mathrm{d}(\mathrm{x})]^{\mathrm{y}}$

$\mathrm{P}_{\mathrm{tb}}(\mathrm{x})=\operatorname{Pr} .\left[\mathrm{d}_{\mathrm{r}}\right]^{\mathrm{y}}$

$\mathrm{P}_{\text {tr }}(\mathrm{x})=\operatorname{Pr} .\left[\mathrm{d}(\mathrm{x})-\mathrm{d}_{\mathrm{r}}\right]^{\mathrm{y}}$

$\mathrm{P}_{\mathrm{tm}}(\mathrm{x})=\mathrm{P}_{\mathrm{tb}}(\mathrm{x})+\mathrm{P}_{\mathrm{tr}}(\mathrm{x})$

End

\subsection{Calculation of power requirement when eNodeB and UE are fixed nodes and RN is movable}

Here also single hop distance between the eNodeB and UE is divided into two parts similar to the previous case. The sensitivity of RN \& UE are assumed to be the same and fixed. However the optimum transmitted power requirement is carried out with the exception that $\mathrm{RN}$ is the moving node where as the eNodeB and UE are fixed nodes unlike previous case.

Algorithm 2: Calculation of optimum power requirement when base station and UE are fixed nodes and Relay node is a movable one 


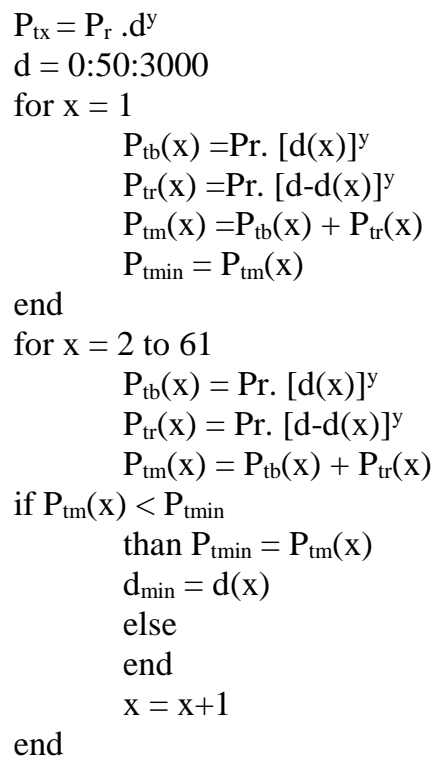

\subsection{Simulation results}

As per Algorithm 1 and Algorithm 2, simulations are carried out with the help of Matlab for both the scenarios. In the first instance, User Equipment is moving where as in the second instance RN is moving with other two nodes remaining fixed. The results obtained from both the simulations are shown in Figure 2 and Figure 3 [10].

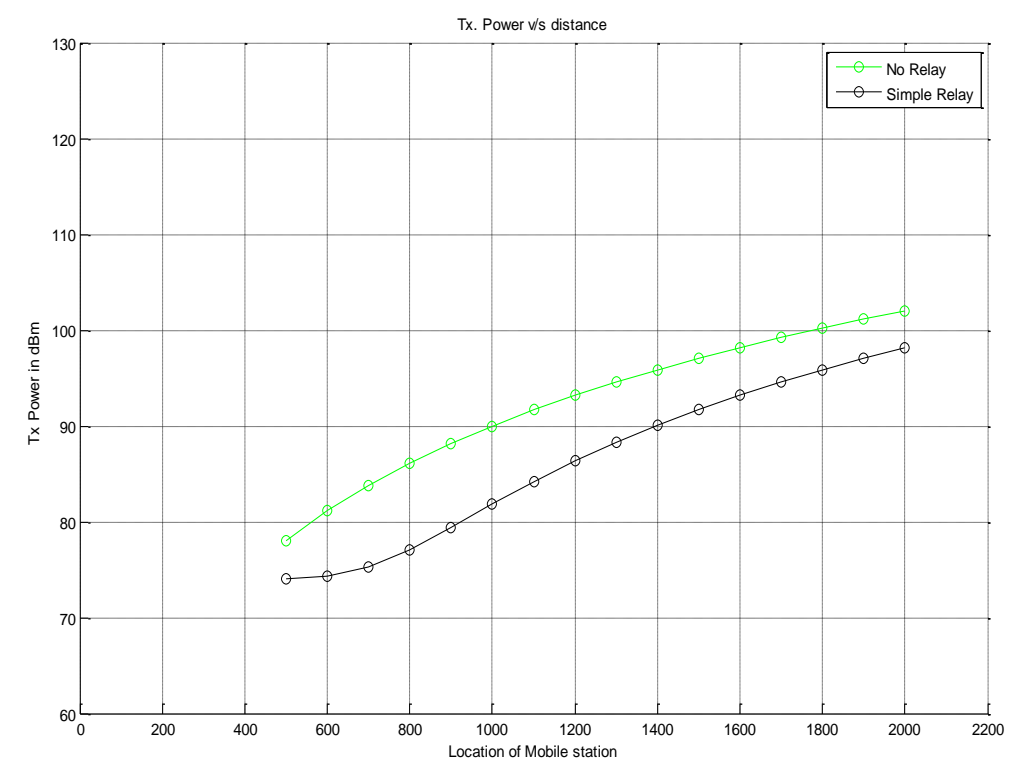

Figure 2. Requirement of transmission power as a function of distance between UE and the base station

From the results of Figure 2 it is clearly evident that requirement of transmitted power decreases after implementation of Relay node irrespective of the location of UE from the Base Station.This concludes a possible extension of coverage resulting from the RN implementation. The result shown in Figure 3 clearly indicates that, requirement of total transmitted power is a function of distance between RN from eNodeB.As the location of RN moves away from $\mathrm{eNodeB}$, transmitted power requirement reduces till the RN reaches the centre between UE and eNodeB.However further movement of RN increases the power requirement. This gives an idea about fixing of Relay nodes in the network to optmize the performance. 


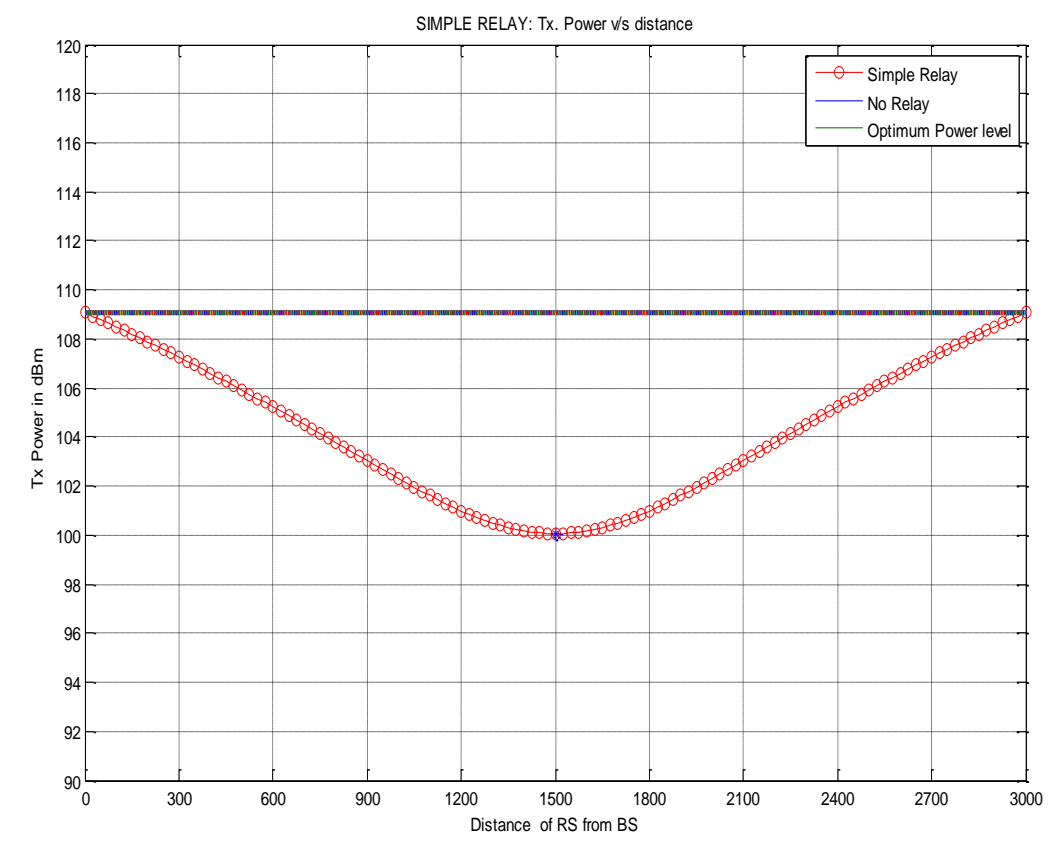

Figure 3. Requirement of transmission power as a function of location of relay from eNodeB

\section{ANALYSIS OF A \& F RELAY UNDER IBL REGIME}

\subsection{Non cooperative environment}

A simple relaying scenario is considered with a Base Station (eNodeB), a Mobile Station (UE), and a Relay node $(\mathrm{RN})$ as shown in Figure 4. The relay is assumed to be an A\&F relay. The link between eNodeB to UE is termed as the direct link, the link between eNodeB and RN is called the backhaul link and the link between RN to UE is termed as relaying link. For transmission of data from eNodeB to UE, First, eNodeB transmits data to the UE using direct link. In second case eNodeB transmits data to the RN using Backhaul link which then forward it to the destination using relaying link.

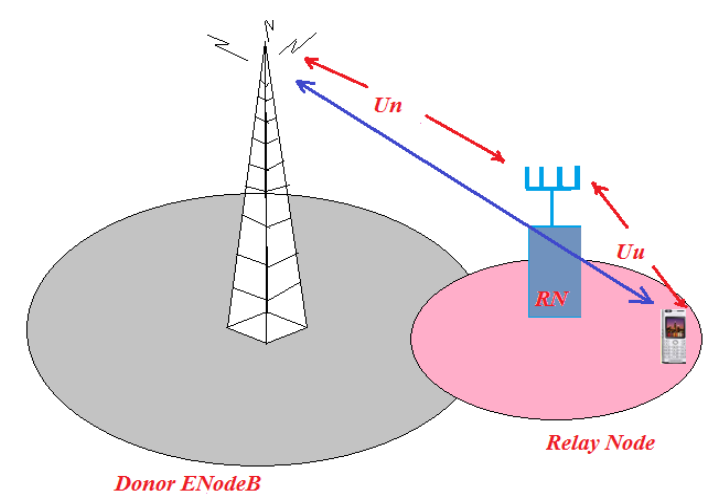

Figure 4. Non cooperative and cooperative relaying

Here we have considered a Gaussian channel with static channel gains $h_{1}, h_{2}$, and $h_{3}$ representing the channel gains (scalars) of the direct link, backhaul link, and relaying link respectively with the corresponding noise vectors denoted by $\mathrm{n}_{1}, \mathrm{n}_{2}$, and $\mathrm{n}_{3}$ respectively. These noise vectors are identically distributed, independent Gaussian vectors: $\mathrm{n} \sim N\left(0, \sigma^{2} \mathrm{I}_{m}\right), \mathrm{n} €\left\{\mathrm{n}_{1}, \mathrm{n}_{2}, \mathrm{n}_{3}\right\}$. Further signals received by UE using direct link and relaying link are $\mathrm{y}_{1}$ and $\mathrm{y}_{3}$ respectively where as signal received at $\mathrm{RN}$ from eNodeB is termed as $\mathrm{y}_{2}, \mathrm{x}$ being the transmitted signal of eNodeB and RN. Let the gain provided by A\&F Relay is G. Thus when no relay is involved, the received signal at the destination using direct path can be denoted as $\mathrm{y}_{1}=h_{1} \mathrm{x}+\mathrm{n}_{1}$ and the received signal-to-noise ratio (SNR) at the UE is given by $\alpha=h_{1}^{2} \cdot \mathrm{x} / \sigma^{2}$. 
Amplification factor of A\&F relay is

$$
\mathrm{G}=\sqrt{\frac{\alpha}{\alpha\left|h_{2}\right|^{2}+1}}
$$

Signal received using direct path without relay is

$$
y_{1}=h_{1} \cdot x+n_{1}^{\beta}
$$

Where

$$
\beta=\sqrt{\frac{1}{\alpha}}
$$

Signal received at $\mathrm{RN}$ from $\mathrm{eNodeB}$ is

$$
y_{2}=h_{2} \cdot x+n_{2} \cdot \beta
$$

Signal at the output of Relay after Amplification is

$$
y_{a}=y_{2} \cdot G
$$

Hence the Signal received at UE from eNodeB with A\&F Relay is

$$
y_{3}=h_{3} \cdot y_{a}+n_{3} \cdot \beta
$$

To findout the effectiveness of RN implementation, SER analysis is carried out at the receiver between signals received by direct path and through A\&F Relay node with the theoretical results [11]. The values of different parameters used in this simulation are indicated in Table 2 as above and the simulation results obtained are shown in Figure 5.

Table 2. Parameters considered for analysis of A\&F relay without cooperative diversity

\begin{tabular}{ll}
\hline \multicolumn{1}{c}{ Parameter } & \multicolumn{1}{c}{ Value } \\
\hline Digital Modulation used & QPSK Modulation \\
Channel type used & AWGN with Rayleigh fading \\
Total transmitted power level & $30 \mathrm{dbm}$ \\
Transmitted power at RN & $27 \mathrm{dbm}$ \\
Transmitted power at eNodeB & $27 \mathrm{dbm}$ \\
\hline
\end{tabular}

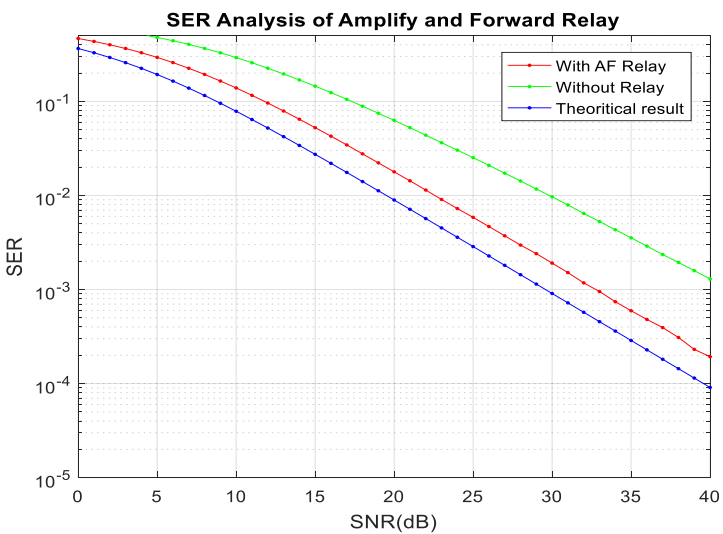

Figure 5. SER Vs SNR curve for non cooperative relay 
Reduction of SER and improvement in the link quality has been noticed after implementation RN. This indicates deployment of relay node improves link throughput and performance.

\subsection{Under cooperative relaying}

Cooperative relaying has emerged as one of the fastest growing research areas for efficient spectrum usage of the wireless networks $[12,13]$. By allowing nodes like mobile handsets and RNs to cooperate among themselves, the communication resources can be utilizes effectively as a result of which improved energy/power efficiency, Cell-edge coverage extension, increased system throughput etc can be achieved by cooperative relaying in comparison to simple relay techniques $[14,15]$.

The output is obtained at the UE by carrying out MRC (maximal ratio combining) of the signals received directly from eNodeB and that via $\mathrm{RN}$ as mentioned in (7) [16]. This is found out for cooperative relaying in continuation with (6). Here y is the signal received by UE after cooperative diversity and $\beta=1 / \sqrt{ } \alpha$.

$$
y=\left(\frac{\overline{h_{1}}}{\beta} y_{1}+\frac{\overline{h_{2}} \cdot \bar{G} \cdot \overline{h_{3}}}{\left(\left|h_{3}\right|^{2}+|G|^{2}+1\right) \cdot \beta}\right) \cdot y_{3}
$$

A comparison is carried out to show the impact of SNR on SER after using cooperative relaying technique. SER is considered to be the performance parameter here for the signals received with and without cooperative relaying [17]. The results obtained are shown in Figure 6.

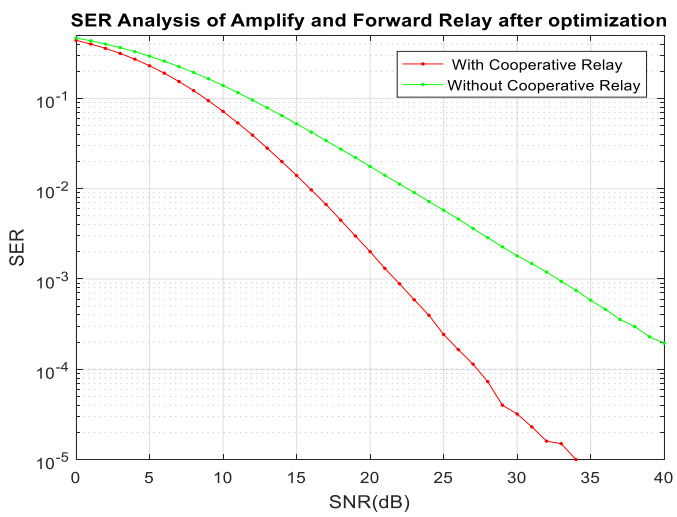

Figure 6. Comparison between SER of A\&F relay under non cooperative environment and co operative environment

The comparative result shown in Figure 6 informs that further reduction of SER can be obtained with the help of cooperative relaying technique. Hence it is possible to improve the system performance by reduction of SER using cooperative relaying concept.

\section{OPTIMIZATION OF COOPERATIVE RELAYING}

In the previous cases, equal power distribution between eNodeB and $\mathrm{RN}$ was assumed. Let us consider an A\&F relay with cooperative diversity. Let the transmit power levels at source be $P_{1}$ and at relay be $P_{2}$ respectively, so that $P_{t}=P_{1}+P_{2}$. Affect of power distribution between eNodeB and RN on the performance is now analysed. To achieve this objective, performance parameter (SER) is now found out by varying the power distribution between eNodeB and RN. Evolutionary optimization algorithm PSO is used for the purpose [18-20].

Algorithm 3: Particle Swarm Optimization Algorithm (PSO) for Relay Optimization.

Input: Fitness function $\mathrm{f}(\mathrm{x})$ is $\mathrm{SER}$, Constraint function $\mathrm{g}(\mathrm{x})$ is $\mathrm{SNR}$, design solution for $\mathrm{x}=\left[\mathrm{P}_{2} / \mathrm{P}_{\mathrm{t}}\right]$ whose value varies between $0 \leq \mathrm{x} \leq 1$.

Output: Optimal value of design parameter $\left(\mathrm{x}_{\mathrm{opt}}\right)$.

Initialize population $(\mathrm{N})$, velocities and positions of all particles randomly.

Specify the boundary limits $\left[0<\mathrm{P}_{2} / \mathrm{P}_{\mathrm{t}}<1\right]$

For each particle 
Compute the fitness function, $\mathrm{f}(\mathrm{x})=[\mathrm{SER}]$

If the fitness value is better than the previous best fitness value ( $\left.\mathrm{P}_{\text {best }}\right)$.

Set $P_{\text {best }}=f(x)$.

\section{End}

Among the group, particle with the best fitness value can be choosen as the $\mathrm{G}_{\text {best. }}$

For every particle

Update particle velocity and position in accordance with (7)

With a condition that the new position should be within upper and lower boundary.

End

Update $\mathrm{P}_{\text {best }}$

Select $\mathrm{G}_{\text {best }}$

End

This process continues until predefined value of iterations is reached or a solution with a fitness value close to the desired value is obtained.

The desired solution achieved is $\mathrm{x}_{\mathrm{opt}}=\mathrm{G}_{\mathrm{best}}$. By Allocating $\mathrm{x}_{\mathrm{opt}}=\left[\mathrm{P}_{2} / \mathrm{P}_{\mathrm{t}}\right]$ to Relay node, design remodelling is done using the new parameters. For this design each swarm consisting of 20 particles runs for 500 iterations. The parameters considered for this algorithm are $w=0.98, c 1=1.7$ and $c 2=1.7$. This PSO algorithm is programmed with MATLAB for evaluation. It is obtained that when $\mathrm{P}_{2} / \mathrm{P}_{\mathrm{t}}=0.73$ optimum results occur. This indicates when $73 \%$ of the total transmitted power is allocated to $\mathrm{RN}$ and $27 \%$ is allocated to eNodeB optimum results are obtained

After optimization, SER analysis of A\&F cooperative relay is again obtained which is shown in Figure 7 in comparison with SER analysis of the same relay without optimization.The results show that reduction of SER and thus improvement is system performance is possible due to optimization.

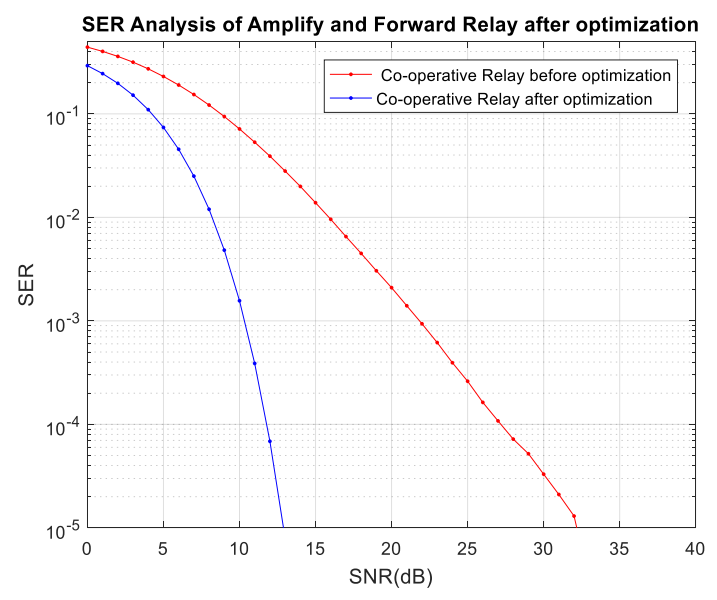

Figure 7. SER analysis of A\&F Relay before and after optimization

\section{CONCLUSION}

This paper presents an overview of relay technology used for the next generation cellular networks. Using experimental method it is concluded that enhancement of coverage and throughput is possible after deployment of Relay nodes where placement of the RN plays a significant role. Further cooperative relaying in comparison to conventional Relaying is discussed for improvement of power efficiency, increased system throughput, cell-edge coverage extension etc which could be very useful even for $5^{\text {th }}$ Generation networks considering their huge power requirement. Then optimization of power is carried out using PSO evolutionary optimization algorithm for performance enhancement of Relays.This work can be extended for obtaining a optimized radio access network in practical scenario with more than one RN using efficient routing, power allocation and scheduling algorithms. Further analysis and optimization of Relays for URLLC networks in the FBL regime can be another research direction in this regard. 


\section{REFERENCES}

[1] A. Abdallah, D. Serhal and K. Fakih, "Relaying Techniques for LTE-Advanced," Proceedings of European Wireless 2015; 21th European Wireless Conference, Budapest, Hungary, pp. 1-6, 2015.

[2] Yifei Yaun, "LTE-Advanced Relay Technology and Standardization," Springer Publication, ISBN 978-3-64229676-5, 2013.

[3] S. P. Padhy, S. Sethi and A. Tripathy, "Performance evaluation of Relays used for next Generation wireless communication networks," 2018 International Conference on Applied Electromagnetics, Signal Processing and Communication AESPC, Bhubaneswar, India, pp. 1-4, 2018

[4] Le Anh Vu, Minh Tran, Van-Duc Phan and Hoang-Nam Nguyen and Thanh-Long Nguyen, "The influence of hardware impairment on the system performance of two-way relaying network," International Journal of Electrical and Computer Engineering IJECE, vol. 9, no. 5, pp. 3623-3632, Oct 2019.

[5] V. Y. F. Tan and M. Tomamichel, "The Third-Order Term in the Normal Approximation for the AWGN Channel," in IEEE Transactions on Information Theory, vol. 61, no. 5, pp. 2430-2438, May 2015.

[6] Padhy Saraju prasad, Panda Madhusmita, Sethi Srinivas and Tripathy Aruna, "Performance Measurement and Optimization of Relays Used for 5G Ultra Reliable Low Latency Communication Network," International Journal of Recent Technology and Engineering IJRTE, vol. 8, no. 5, pp. 5017-5023, 2020.

[7] A. Krendzel, "LTE-A Mobile Relay Handling: Architecture Aspects," European Wireless 2013; 19th European Wireless Conference, Guildford, UK, pp. 1-6, 2013.

[8] S. Baroudi and Y. R. Shayan, "Performance enhancement of multi-hop wireless systems by optimizing the number and location of relays," International Wireless Communications and Mobile Computing Conference (IWCMC), Dubrovnik, pp. 465-468, 2015.

[9] Y. Hu, J. Gross and A. Schmeink, "QoS-Constrained Energy Efficiency of Cooperative ARQ in Multiple DF Relay Systems," in IEEE Transactions on Vehicular Technology, vol. 65, no. 2, pp. 848-859, Feb 2016.

[10] Dr Houman Zarrinkoub, "Understanding LTE with Matlab: From Mathematical Modeling to Simulation and Prototyping” John Wiley \& Sons Ltd, United Kingdom, ISBN: 9781118443415, 2014.

[11] J. N. Laneman, D. N. C. Tse and G. W. Wornell, "Cooperative diversity in wireless networks: Efficient protocols and outage behavior," in IEEE Transactions on Information Theory, vol. 50, no. 12, pp. 3062-3080, Dec. 2004.

[12] Tan N. Nguyen, Van-Duc Phan, Hoang-Nam Nguyen, Minh Tran and Tran Thanh Trang, "Performance analysis for power-splitting energy harvesting based two-way full-duplex relaying network over nakagami-m fading channel," Telecommunication, Computing, Electronics and Control TELKOMNIKA, vol. 17, no. 4, pp.1595-1603, Aug 2019.

[13] Dinh-Thuan Do, Chi-Bao Le, Hong-Nhu Nguyen, Tam Nguyen Kieu, Si-Phu Le, Ngoc-Long Nguyen, Nhat-Tien Nguyen and Miroslav Voznak, "Wireless power transfer enabled NOMA relay systems: two SIC modes and performance evaluation" Telecommunication, Computing, Electronics and Control TELKOMNIKA, vol. 17, no. 6, pp. 2697-2703, Dec 2019.

[14] M. F. Kader, M. B. Shahab and S. Y. Shin, "Exploiting Non-Orthogonal Multiple Access in Cooperative Relay Sharing," in IEEE Communications Letters, vol. 21, no. 5, pp. 1159-1162, May 2017.

[15] Q. Li, R. Q. Hu, Y. Qian and G. Wu, "Cooperative communications for wireless networks: techniques and applications in LTE-advanced systems," in IEEE Wireless Communications, vol. 19, no. 2, pp. 22-29, Apr 2012.

[16] Van-Duc Phan, Tan N. Nguyen, Minh Tran and Tran Thanh Trang, "Outage probability analysis of dual energy harvesting relay network over rayleigh fading channel using SC and MRC technique," Indonesian Journal of Electrical Engineering and Computer Science IJEECS), vol. 16, no. 2, pp. 803-811, Nov 2019.

[17] Duong Huu Ai, Van Loi Nguyen, "BER analysis of amplify-and-forward relaying FSO systems using APD receiver over strong atmospheric turbulence channels," International Journal of Electrical and Computer Engineering IJECE, vol. 9, no. 5, pp. 3678-3686, Oct 2019.

[18] Panda M, Patnaik S K, Mal A K, "Performance enhancement of a VCO using symbolic modelling and optimization," IET Circuits, Devices \& Systems, vol. 12, no. 2, pp. 196-202, 2018.

[19] Panda M, Patnaik S K, Mal A K, Ghosh S "Fast and optimised design of a differential VCO using symbolic technique and multi objective algorithms," IET Circuits, Devices \& Systems, vol. 13, no. 8, pp. 1187-1195, 2019.

[20] Kalyanmoy Deb, J. Sundar, "Multi-objective optimization using evolutionary algorithms," GECCO '06: Proceedings of the 8th annual conference on Genetic and evolutionary computation, pp. 635-642, 2006.

\section{BIOGRAPHIES OF AUTHORS}

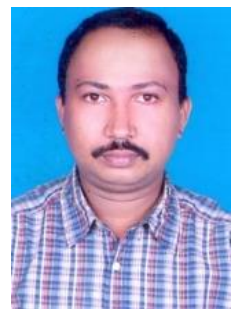

Saraju Prasad Padhy obtained his Master in Engineering degree from BIT, Mesra, India with a specialization in Microwave Systems. He has more than 22 years of industrial experience in the field telecommunication both in India and abroad. He worked in different wireless communication technologies starting from CDMA, GSM to Wi-MAX and LTE Advanced. His subject interests include communications, signal processing and wireless communication networks. He is presently perusing Ph.D. in the field of wireless communication. He has already published some papers on next generation wireless networks in reputed journals and conferences. 

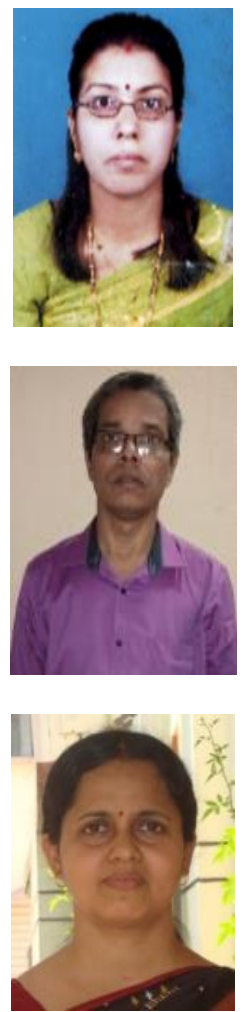

Madhusmita Panda obtained her MTech from BPUT, Rourkela, India with a specialization in Microelectronics and VLSI design. She has published about 10 papers in different national and international conferences and journals. She has academic experience of about 15 years during which she taught several subjects to both UG and PG students. Her subject interests include Semiconductor devices and circuits. Presently, she is serving as an Assistant Professor in the department of Electronics and Communication Engineering at ITER (Institute of Technical Education and Research) under SOA University, Bhubaneswar in addition to perusing her Ph.D. in the field of Microelectronics.

Srinivas Sethi obtained his Ph.D. from Berhampur University, Berhampur, India in the field of Computer Science. He has published about 60 papers in different national and international conferences and journals. He also authored three text books apart from successfully supervising $5 \mathrm{Ph}$.D. scholars and 15 masters' degree students. He was involved with more than 35 International/ National Level Conferences/Seminars /Workshops in the capacity of Special session Chair /Coordinator/Technical Committee Member/ Advisor Committee Member. He also organized 5 State Level Conferences / Seminars /Workshops and delivered more than 50 Invited Lectures at International/National/University level Conferences/Seminars.

Aruna Tripathy obtained her Ph.D. from IIT, Kharagpur, India with a specialization in Wireless communications. She has published about 40 papers in different national and international conferences and journals. She has both an industrial and academic experience of about 25 years. She has taught several subjects to both UG and PG students.She also authored a text book "Optoelectronics and Systems" published by Studium Press (Int). About 33 master's theses have been supervised by her. Her subject interests include communications, signal processing and photonics. Presently, she is serving as the head of the department of Instrumentation and Electronics Engineering at college of Engineering and Technology (CET), Bhubaneswar. 